\title{
Aplicando o modelo de regressão linear para determinar as horas de aprendizagem: um estudo de caso
}

\author{
Paulo H. L. Silva (UniCarioca)
}

\section{Resumo}

Este artigo apresenta, de forma prática, a aplicação dos conceitos da regressão linear simples para estimar a quantidade de horas de estudo destinadas para candidatos à certificação ITIL ${ }^{\oplus}$ (Information Technology Infrastructure Library) V3 Foundation. Muitos candidatos não sabem qual o momento certo para a realização do exame. Alguns centros de treinamento avaliam algo entre $85 \%$ e $90 \%$ de acertos nos simulados para se ter a aprovação "garantida". Ter uma estimativa de quantas horas de estudo são necessárias para obter tal desempenho ou se um curso irá ajudar a obter a certificação é o foco deste estudo de caso. O modelo proposto foi aplicado a dois grupos distintos de candidatos que prestaram o exame de certificação, mas apenas um dos grupos realizou um curso preparatório oferecido por um grande centro de treinamento, enquanto o outro grupo estudou por meio de livros, apostilas e simulados disponíveis pelos mais diversos meios (físico e/ou digital). O modelo aplicado mostrou notória confiabilidade, demonstrando que o que realmente influi é a quantidade de horas destinadas ao estudo, independentemente do candidato ter realizado um curso preparatório para certificação.

Palavras-Chave: regressão linear; ITIL ${ }^{\oplus}$; exame de certificação; tecnologia da informação.

\begin{abstract}
This paper presents a practical way to apply the concepts of simple linear regression to estimate the amount of study hours dedicated to certification candidates to ITIL (Information Technology Infrastructure Library) V3 Foundation. Many candidates don't know what the correct time for the exam. Some training centers talk somewhere between $85 \%$ and $90 \%$ correct in simulated to be approved "guaranteed". However, have an estimate of how many hours of study are required to obtain this performance, or if a course will help you get certified, is the focus of this case study. The proposed model was applied to two different groups of candidates who took the certification exam, but only one group held a preparatory course offered by a well-known training center, while the other group studied through books, handouts and simulated available by variety of means (physical and / or digital). The applied model showed remarkable reliability, demonstrating that what really affects is the amount of hours devoted to the study, regardless of the candidate has carried out a preparatory course for certification.
\end{abstract}

Keywords: linear regression; ITIL ${ }^{\circledR}$ certification exam; information technology.

\section{Introdução}

A grande demanda por profissionais qualificados no Brasil já é uma realidade há alguns anos (Filho, 2007). Não seria diferente com relação aos profissionais de Tecnologia da Informação. Hoje em dia, uns dos maiores ativos que as organizações dispõem são as suas informações (Meneguelli e Souza, 2007). As certificações em governança de Tecnologia da Informação são uma excelente opção para quem quer se diferenciar nesse mercado novo e dinâmico. A certificação ITIL ${ }^{\circledR}$ (Information Technology Infrastructure Library) V3 fornece aos profissionais de Tecnologia da Informação os conhecimentos e as ferramentas para estar um passo à frente na prestação de serviços ou na área de apoio, em todos os componentes: manutenção, gestão e execução (Figura 1). 


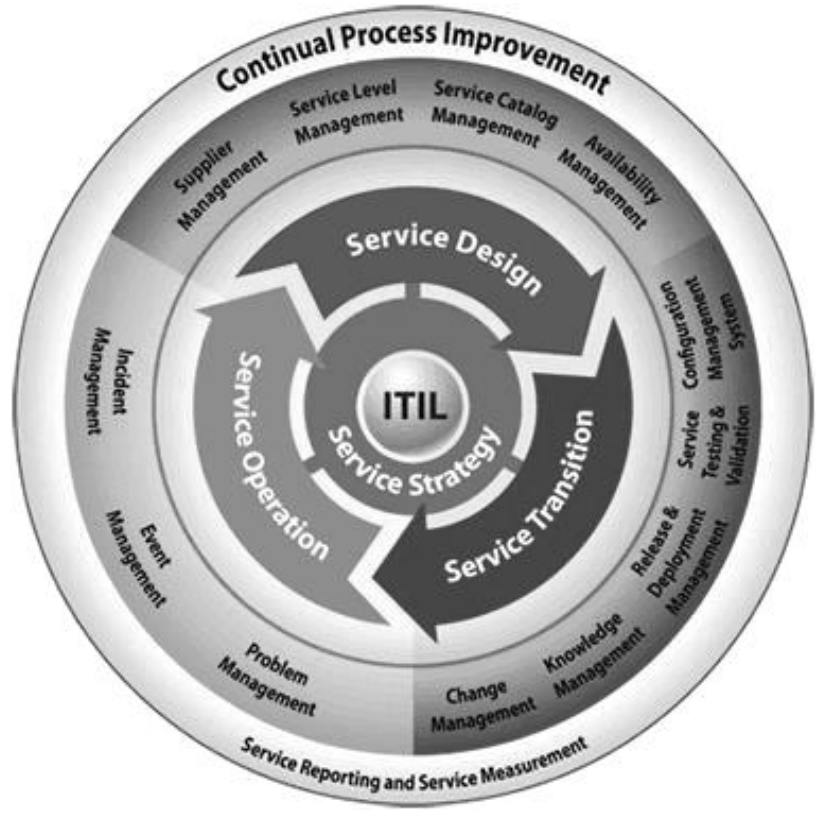

Figura 1 - ITIL ${ }^{\oplus}$ Processos. (Fonte: http://ctclc.com/courses/itil/itildesc.htm)

As últimas descobertas de grandes reservas de petróleo offshore no pré-sal geraram uma concorrência em nível mundial e colocaram o Brasil entre as nações com maiores reservas provadas, com 14,2 bilhões de barris (BP, 2011, p.6). Cada vez mais os sistemas informacionais são utilizados para simular a realidade a partir de dados preliminares distribuídos nos campos de produção de petróleo. Portanto, a validade dos resultados deve, além de atender certos requisitos, ser certificada com base nas melhores práticas de gestão (Chermont, 2001).

Logo, para que os serviços prestados atendam a uma qualidade de nível internacional devem passar por uma administração altamente profissional e afinada com as melhores práticas em gestão. Na área de Tecnologia da Informação, as melhores práticas foram agrupadas, de modo que podem ser encontradas na ITIL ${ }^{\oplus}$ (Estratégia de Serviço (ES), Desenho de Serviço (DS), Transição de Serviço (TS), Operação de Serviço (OS), Melhoria de Serviço Continuada (MSC)) (Rance, 2007, p. 2 ).

A ITIL ${ }^{\oplus}$ é mundialmente reconhecida como um conjunto das melhores práticas de serviços de Tecnologia da Informação (IT Service Management - ITSM). A ITIL ${ }^{\oplus}$ foi publicada no final dos anos 80 no Reino Unido, em nome da Agência Central de Comunicações e Telecomunicações (CCTA) agora incluída no âmbito do Gabinete do Comércio do Governo (OGC). Inicialmente, seu uso foi limitado ao Reino Unido e Holanda. A segunda versão do ITIL $^{\oplus}$ foi publicada como um conjunto de livros-revista entre 2000 e 2004. É o framework mais aceito no mundo para o gerenciamento de serviços de TI. Fornece um conjunto coeso de orientações de melhores práticas extraídas dos setores públicos e privado em todo o mundo. Recentemente, em 2011, sofreu uma atualização (Rance, 2007, p.8).

Segundo Arraj (2010, p.4), a ITIL ${ }^{\oplus}$ tem sido adotada por centenas de organizações, que incluem Microsoft, IBM, Barclays Bank, HSBC, Guinness, Procter \& Gamble, British Airways, Hewlett Packard, NASA, Disney, etc. Além disso, a ITIL ${ }^{\oplus}$ pode ser usada em conjunto com outras boas práticas para a gestão da qualidade e gestão de Tecnologia da Informação, tais como COBIT (um framework de Tecnologia da Informação de Governança e controles), Seis Sigma (uma metodologia de qualidade), TOGAF (um quadro de arquitetura de Tecnologia da Informação) e ISO 27000 (um padrão para segurança de Tecnologia da Informação). 
Ainda para Arraj (2010, p.4), a ITIL fornece uma abordagem sistemática e profissional para a gestão da prestação de serviços de Tecnologia da Informação. Adotar a sua orientação oferece aos usuários uma gama enorme de benefícios que incluem, entre outros:

- Redução de custos;

- Padrões e direcionamentos,

- $\quad$ Aumento da satisfação do cliente;

- Melhoraria da produtividade;

- Utilização de uma linguagem comum;

- Melhor utilização de habilidades e experiência e

- Melhoria dos serviços de Tecnologia da Informação por meio do uso de melhores práticas comprovadas.

Para se conseguir a certificação do nível Foundation não é obrigatório fazer um curso nem comprovar experiência na área de gerenciamento de serviços de Tecnologia da Informação. A prova é realizada online, podendo ser feita em qualquer cidade que tenha um centro de testes VUE ou PROMETRIC. Por ser o nível mais acessível e o primeiro da pirâmide de certificações, o nível Foundation é o mais procurado, até mesmo porque sem esse nível o profissional não poderá galgar os demais (Figura 02).

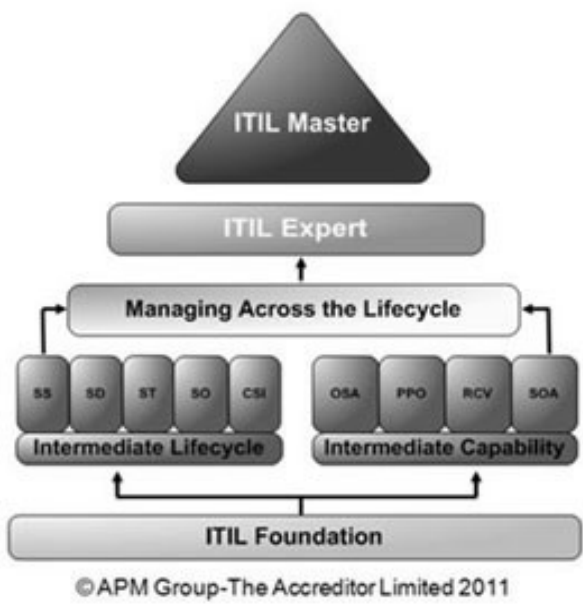

Figura 2 - Níveis de qualificação. Fonte: http://www.itil-officialsite.com/ Qualifications/ITILQualificationScheme.aspx

\section{Materiais e Métodos}

\section{Regressão linear simples}

Conforme Wooldridge (2009, p.22), Lewis-Beck (1980) e Milone e Angelini (1995, p.84), a regressão linear simples pode ser empregada para observar a relação entre duas variáveis de um processo. A análise de regressão incide na averiguação de uma análise estatística com a finalidade de constatar a existência de um relacionamento entre uma variável chamada dependente, a que se deseja prever, e outras variáveis chamadas independentes, utilizadas para se fazer a previsão. Além de obter valores para a variável dependente, a 
análise de regressão permite identificar o tipo de relação matemática existente entre as variáveis dependente e independente.

Para Werkema e Aguiar (1996, p.12), a análise de regressão pode ser utilizada quando se quer prever, descrever, controlar ou estimar a variável de interesse em função das variáveis que a influenciam.

O modelo estatístico para a análise de regressão seria (Wooldridge, 2009, p.22):

$$
\mathrm{Yi}=\beta 0+\beta 1 \mathrm{Xi}+\mathcal{E} \mathrm{i}(\text { eq. } 1)
$$

Onde:

- $\quad \mathrm{Yi}$ = valor da variável dependente $\mathrm{Y}$, quando o valor da variável independente $\mathrm{X}$ assumir o i-ésimo nível;

- $\quad \beta 0=$ constante de regressão. Representa a média aritmética do valor Y quando X é igual a 0 . Intercepto de Y;

- $\quad \beta 1=$ coeficiente de regressão. Representa a variação esperada de Y por unidade de variação de X;

- $\quad \mathrm{Xi}=\mathrm{i}$-ésimo nível da variável independente $(\mathrm{i}=1,2,3, \ldots, \mathrm{n})$;

- $\quad \varepsilon i=$ erro aleatório em Y para a observação i, melhor visto na Figura 03.

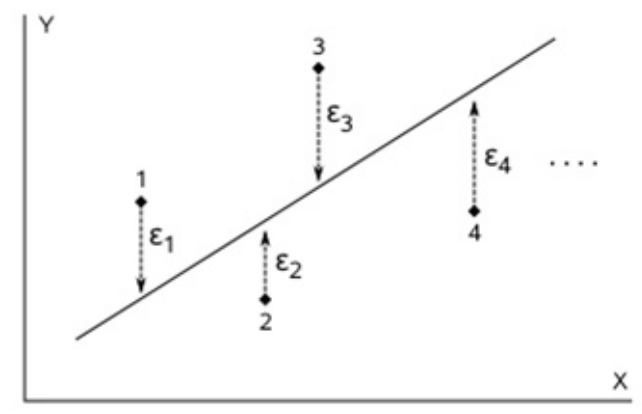

Figura 03 - Erros na Regressão Linear Simples. Fonte: http://www.mspc. eng.br/matm/prob_est500.shtml

\section{O Método dos Mínimos Quadrados (MMQ)}

Segundo a equação 02, temos a curva linearizada, que é chamada de linha de previsão:

$$
\hat{\mathrm{Y}} \mathrm{i}=\mathrm{b} 0+\mathrm{b} 1 \mathrm{Xi}(\text { eq. } 2)
$$

- $\quad \mathrm{i}=$ valor previsto de $\mathrm{Y}$ para a observação $\mathrm{i} ;$

- $\quad \mathrm{Xi}=$ valor de $\mathrm{X}$ para a observação $\mathrm{i}$;

- $\quad$ b0 = intercepto da amostra, e;

- $\quad$ b1 = inclinação da amostra.

Segundo Kutner (2005, p.15), é o método que minimiza a soma das diferenças, elevadas ao quadrado, entre os valores verdadeiros (Yi) e os previstos ( $\hat{Y} i)$, utilizando a equação da regressão linear simples. Essa soma de diferenças elevadas ao quadrado é igual a: 


$$
\begin{gathered}
\sum_{i=1}^{n}=\left[\left(Y_{i}-\hat{Y}_{i}\right)\right]^{2} \\
\text { Temos que } \hat{Y}_{i}=b_{0}+b_{1} X_{i}: \\
\sum_{i=1}^{n}[(\mathbf{Y i}-\hat{\mathbf{Y}} \mathbf{i})]^{2}=\sum_{i=1}^{n}\left[(\mathbf{Y i}-(\mathbf{b} \mathbf{0}+\mathbf{b} \mathbf{1} \mathbf{X} \mathbf{i})]^{2}\right.
\end{gathered}
$$

O MMQ determina os valores de b0 e b1 que minimizam a soma das diferenças ao quadrado. Quaisquer outros valores de b0 e b1 que não sejam determinados por esse método resultarão em uma soma maior das diferenças elevadas ao quadrado entre os valores reais (Yi) e os valores previstos ( $(\hat{\mathrm{i}})$.

Cálculo de b1, b0 e Intercepto de Y

Simplificando a fórmula para calcular a inclinação b1 proposta por Kutner (2005, p.17), teremos que:

$$
b_{1}=S Q X Y / S Q X \quad \text { (eq. 5) }
$$

$$
\begin{aligned}
& \text { Em que: } \\
& \qquad \begin{aligned}
\mathrm{SQXY}= & \sum_{i=1}^{n}(X i-\bar{X})(Y i-\bar{Y}) \\
\mathrm{SQX}= & \sum_{i=1}^{n}(X i-\bar{X})^{2} \\
& \bar{X} \text { e } \bar{Y}=\mathrm{X} \text { médio e } \mathrm{Y} \text { médio, respectivamente. }
\end{aligned}
\end{aligned}
$$

Alguns pressupostos devem ser atendidos antes de utilizarmos tais equações. São eles:

- Linearidade - a relação entre as variáveis é linear;

- Independência de erros - os erros $\mathcal{E}$ i são independentes entre si;

- Normalidade dos erros - os erros $\mathcal{E}$ i são distribuídos normalmente para cada valor de X e

- Igualdade das variâncias - a variância dos erros $\mathcal{E}$ i é constante para todos os valores de X, ou seja, a variabilidade dos valores de $\mathrm{Y}$ é a mesma quando $\mathrm{X}$ é um valor muito baixo ou elevado.

\section{O Coeficiente de Determinação}

Segundo Kutner (2005, p. 74), o coeficiente de determinação mede a proporção da variação em Y, que é explicada pela variável independente $\mathrm{X}$ no modelo de regressão. Quanto mais perto de 1 for essa relação, mais forte é a relação entre as variáveis e menor a variabilidade e isso depende de outros fatores.

Segundo a fórmula a seguir, temos: 


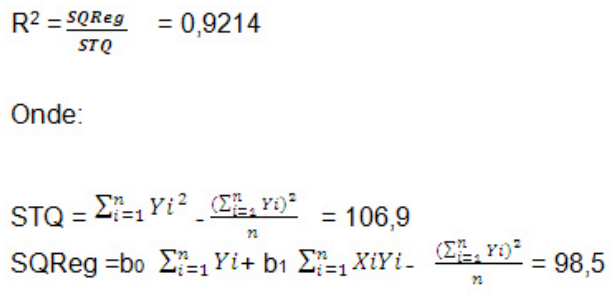

A metodologia desenvolvida para este estudo teve início com a distribuição de um simples mapa de resultados, em que os candidatos marcaram a quantidade de horas de estudos até um dado simulado e o resultado do referido simulado. Após essa parte da coleta, os dados foram tabelados e apresentados na seção seguinte (Item Resultados).

\section{Resultados}

Um grupo de 10 candidatos foi selecionado para concretizar esse estudo, e as medidas foram feitas de diversas formas. Um grupo de 5 candidatos estudou por conta própria por meio de apostilas e simulados; também se formou um grupo de estudo para tirar dúvidas e apresentar novas questões, denominado GRUPO 01. Outro grupo de 5 candidatos realizou um curso de 16 horas em um centro de treinamento de renome e utilizou apenas o material fornecido por ele, denominado GRUPO 02.

O GRUPO $01 \mathrm{fez}$ um simulado de 5 em 5 horas de estudo até completar, no mínimo, 20 horas de estudo, enquanto o GRUPO $02 \mathrm{fez} \mathrm{um} \mathrm{simulado} \mathrm{após} \mathrm{cada} \mathrm{período} \mathrm{de} 8$ horas, ou 1 dia de curso. Depois, os candidatos do GRUPO 02 fizeram uma revisão de, no mínimo, mais 2 horas, como recomenda o treinamento.

A prova de certificação do nível Foundation tem 40 questões. Para ser aprovado, o candidato precisa de, no mínimo, 65\% de acertos, ou seja, para ser aprovado o candidato precisa acertar, pelo menos, 26 questões. O exame tem 1 hora de duração e pode ser realizado em português. Para simplificar, vamos trabalhar com a quantidade de Erros (E) que os candidatos podem ter no caso estudado. Assim, os candidatos podem errar até 14 questões para obter a aprovação.

Como mencionado anteriormente, foram colhidas as informações de 2 grupos (GRUPO 1 e GRUPO 2). Os resultados dos simulados, no decorrer das horas estudadas, estão apresentados na Tabela 01:

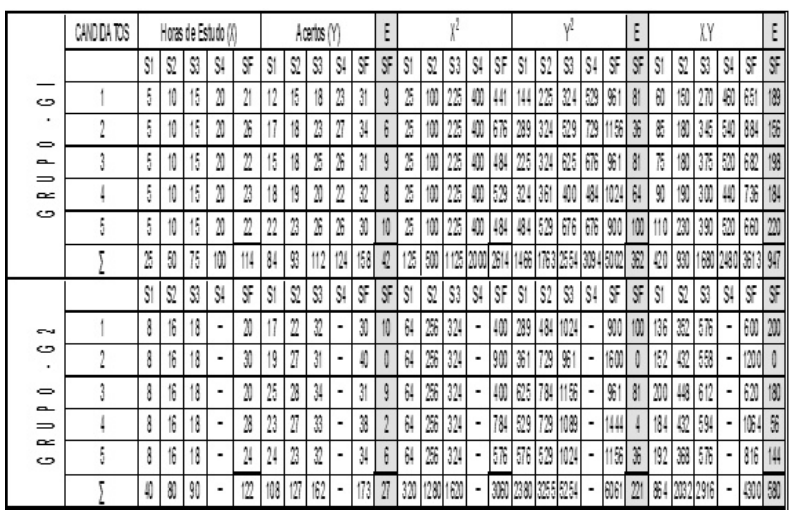

Tabela 01 - Resultados simulados. Fonte: autor. 
Onde “S1, S2, S3, S4 e SF” são, respectivamente, acertos no Simulado 01, 02, 03, 04 e Final. Para o GRUPO 2, foram apenas 3 simulados antes do Simulado Final (SF). "E” para a quantidade de erros no Simulado Final.

A coluna Horas de Estudo representa que, para o Grupo 01, no Simulado 1, os candidatos estudaram 5 horas; para o Simulado 2, totalizaram 10 horas de estudo; para o Simulado 3 contabilizaram 15 horas e para o Simulado 4 fecharam em 20 horas. Para o Simulado Final uma revisão de caráter individual foi feita. Para o Grupo 02, o centro de treinamento aplicou simulados a cada 8 horas de estudo, sendo feita uma revisão de 2 horas para o Simulado 3, e cada um realizou uma revisão, de acordo com a sua necessidade, para o Simulado Final.

Utilizando as equações 2, 5 e 6 a partir dos dados dos Simulados Finais, podemos obter as seguintes equações de previsões da Tabela 02:

\begin{tabular}{|c|c|c|c|}
\hline Equaçäo & Valores & Valores & Valores \\
\hline $\mathrm{b}_{1}$ & $-0,72$ & $-0,95$ & $-0,97$ \\
\hline $\mathrm{SQXY}$ & $-10,6$ & $-78,8$ & $-101,4$ \\
\hline $\mathrm{SQX}$ & 14,8 & 83,2 & 104,4 \\
\hline$\overline{\mathrm{Y}}$ & 8,4 & 5,4 & 6,9 \\
\hline$\overline{\mathrm{X}}$ & 22,8 & 24,4 & 23,5 \\
\hline $\mathrm{b}_{\mathbf{2}}$ & 24,7 & 28,5 & 29,5 \\
\hline
\end{tabular}

Tabela 02 - Elaboração das equações de previsão. Fonte: autor.

De acordo com as 3 equações apresentadas no final da tabela acima (Tabela 02) pode-se concluir que são necessárias aproximadamente 16 horas de estudo para um candidato ser aprovado no nível Foundation, independentemente do candidato ter realizado um curso em um centro de treinamento ou estudado por conta própria. O diferencial entre fazer um curso num centro de treinamento e estudar por conta própria é que a média de acertos dos candidatos que realizaram um curso tende a ser maior. No entanto, a realização de um curso não é imprescindível para aprovação. Vale muito mais a dedicação aos estudos do candidato.

Deve-se lembrar que as equações são baseadas no número de erros que o candidato pode ter, no máximo, 14 erros. Portanto, em nenhuma das equações tal limite é atingido, pois nas equações para um mínimo de 16 horas os resultados seriam:

- $\quad$ Para o GRUPO 1: $\hat{\mathrm{Y}} \mathrm{i}=24,7-0,72 \mathrm{Xi}=>\hat{\mathrm{Y}} \mathrm{i}=24,7-0,72 .(16)=13,18$ erros.

- $\quad$ Para o GRUPO 2: $\hat{Y} i=28,5-0,95 X i=>\hat{Y} i=28,5-0,95 .(16)=13,3$ erros.

- $\quad$ Para os 10 candidatos: $\hat{Y} i=29,5-0,97 X i=>\hat{Y} i=29,5-0,97 .(16)=13,98$ erros.

Portanto, pode-se unificar os dois grupos a partir da equação da previsão para os 10 candidatos e montar nlova tabela (Tabela 03), com a seguinte previsão geral de quantidade de horas de estudo baseada na quantidade de erros:

\begin{tabular}{|c|c|c|c|c|c|c|c|}
\hline Horas de Estudo $(\mathrm{X})$ & 0 & 5 & 10 & $\mathbf{1 5}$ & $\mathbf{2 0}$ & 25 & 30 \\
\hline$\hat{\mathrm{Y}}$ (previsão de erros) & 29.5 & 24.6 & 19.8 & 14.9 & 10.1 & 5.22 & 0.36 \\
\hline
\end{tabular}

Tabela 03 - Resultados dos erros previstos em função das horas de estudo. Fonte: autor. 


\section{Discussão}

O modelo proposto cumpre o seu papel de aferir que o resultado não depende de outras variáveis além da quantidade de horas de estudo no resultado final. É de grande importância por parte dos futuros candidatos uma breve análise na decisão de se inscrever em um treinamento em um grande centro, pois tal decisão acarretará custos adicionais, muito acima do processo de estudo individual/coletivo como o Grupo 1 se propôs a fazer.

Tais resultados podem ser comparados com o recomendado pela própria entidade que administra a ITIL ${ }^{\circ}$. Segundo o Plano de Ensino (2010, p.3), são recomendadas 18 horas de estudo.

No modelo proposto neste estudo pela regressão linear, agrupando todos os dez candidatos, caso algum candidato estude 18 horas, ou seja, a quantidade de horas de estudo referenciada anteriormente, teremos:

$$
\hat{Y} i=29,5-0,97 \times 18=12,04 \text { erros }
$$

Como não existem 12,34 erros, poderíamos dizer que o candidato teve 13 erros, ou seja, o candidato seria aprovado com 27 questões certas. Para ratificar a acurácia do modelo, podemos calcular o seu Coeficiente de Determinação, também chamado de $\mathrm{R}^{2}$, é uma medida de adequação de um modelo estatístico linear generalizado. $\mathrm{O}$ valor de $\mathrm{R}^{2}$ varia entre 0 e 1 , indicando, em percentagem, o quanto o modelo consegue elucidar os valores observados. Quanto maior o $\mathrm{R}^{2}$, mais explicativo é modelo e melhor ele se ajusta à amostra (Triola,1999).

Cálculo do Coeficiente de Determinação (eq. 07):

$$
\mathrm{R}^{2}=\frac{\text { SQReg }}{\text { STQ }}=0,9214
$$

Onde:

$$
\begin{aligned}
& \mathrm{STQ}=\sum_{i=1}^{n} Y i^{2}-\frac{\left(\sum_{i=1}^{n} Y_{i}\right)^{2}}{n}=106,9 \\
& \mathrm{SQReg}=\mathrm{b}_{0} \sum_{i=1}^{n} Y i+\mathrm{b}_{1} \sum_{i=1}^{n} X i Y i-\frac{\left(\sum_{i=1}^{n} Y i\right)^{2}}{n}=98,5
\end{aligned}
$$

Portanto, 92\% da variação nos erros podem ser explicadas pela variabilidade nas horas de estudo. Esse valor para R2 indica uma forte relação linear positiva entre as duas variáveis, uma vez que a utilização de um modelo de regressão reduziu em $92 \%$ a variabilidade na previsão dos erros no exame. Somente $8 \%$ da variabilidade da amostra, em termos de erros, são decorrentes de outros fatores que não aqueles que são considerados pelo modelo de regressão linear que utiliza as horas de estudo.

Portanto, conclui-se que uma preparação em um centro de treinamento não influi fortemente no modelo. A preparação do candidato, o esforço de cada um pela busca do conhecimento e da própria certificação é o fator de maior importância e impacto do modelo apresentado. 


\section{Referências Bibliográficas}

ARRAJ, V. ITIL ${ }^{\otimes}:$ The Basics. United Kingdom, 2010. Disponível em:http://www.best-management- practice.com/gempdf/ITIL_The_ Basics.pdf - acessado em 02 de fev. de 2014, 5p

BP. BP Statistical Review of World Energy. London, UK, 2011. Disponível em: bp.com/statisticalreview - acessado em 05 fev. 2014 , $19 p$.

CHERMONT, G. S. A Qualidade na Gestão de Projetos de Sistemas de Informação. Dissertação (Mestrado em Programa de Engenharia de Produção - COPPE) Universidade Federal do Rio de Janeiro. RJ, 2001.

FILHO, A. M.S. - Capital Humano: necessidade essencial às empresas e diferencial para competitividade. Revista Espaço AcadêmiCO, 75, 2007

KUTNER, M.H. et al. Applied Linear Statistical Models - Fifth Edition. McGraw-Hill, New York, NY (United States), 2005.

LEWIS-BECK, M.. Applied Regression: an introduction. Series Quantitative Applications in the Social Sciences. SAGE University Paper, 1980.

MENEGUELLI, D.; SOUZA, D. B. L. MENSURÁVEL X INTANGÍVEL: O desafio da mensuração dos resultados na Gestão do Conhecimento. Estação Científica Online (Juiz de Fora), 4. 2007.

MILONE, G.; ANGELINI, F. Estatística Aplicada. São Paulo: Atlas, 1995.

PRÁTICAS ITIL PARA GERENCIAMENTO DE SERVIÇO - Certificado de Fundamentos da ITIL V3 em Gerenciamento de Serviço de TI PLANO DE ENSINO - United Kingdom, 2010.

RANCE, S. et al. An Introductory Overview of ITIL ${ }^{\circledR}$ V3. United Kingdom, 2007. Disponível em: http://www.best-management-practice.com/gempdf/itSMF_An_Introductory_Overview_of_ITIL_V3.pdf - acessado em 02 de fev. de 2014, 58p.

TRIOLA, Mario F. Introdução à Estatística. Rio de Janeiro: LTC, 1999.

WERKEMA, M. C. C.; AGUIAR, S. Análise de Regressão: como entender o relacionamento entre as variáveis de um processo - $1^{a}$ Edição. Belo Horizonte - MG, 1996.

WOOLDRIDGE, J. M. Introductory Econometrics - A Modern Approach - Fourth Edition. Cengage Learning, Mason, OH - United States, p.22-35, 2009.

\section{Agradecimentos}

O autor agradece a todos os candidatos que enviaram os resultados dos simulados e de aprovação na certificação. Também agradeço à UniCarioca a oportunidade de publicar este estudo de caso. 